\title{
Auger electron and photoabsorption spectra of glycine in the vicinity of the oxygen K-edge measured with an X-FEL
}

\author{
A. Sanchez-Gonzalez ${ }^{1}$, T. R. Barillot ${ }^{1} \ddagger$, R. Squibb ${ }^{2}$, P. \\ Kolorenč $^{3}$, M. Agaker ${ }^{4}$, V. Averbukh ${ }^{1}$, M. J. Bearpark ${ }^{5}$, \\ C. Bostedt ${ }^{6}$, J. D. Bozek ${ }^{7}$, S. Bruce ${ }^{8}$, S. Carron \\ Montero $^{6}$, R. N. Coffee $^{6}$, B. Cooper $^{1}$, J. P. Cryan $^{9}$, M. \\ Dong $^{4}$, J. H. D. Eland ${ }^{10}$, L. Fang ${ }^{8}$, H. Fukuzawa ${ }^{11}$, M. \\ Guehr $^{9}$, M. Ilchen ${ }^{12}$, A. S. Johnsson ${ }^{1}$, C. Liekhus-S ${ }^{9,13}$, \\ A. Marinelli ${ }^{6}$, T. Maxwell ${ }^{6}$, K. Motomura ${ }^{11}$, M. Mucke ${ }^{4}$,

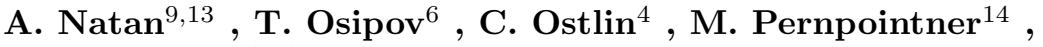 \\ V. S. Petrovic ${ }^{9,13}$, M. A. Robb ${ }^{5}$, C. Sathe $^{1} 5$, E. R. \\ Simpson $^{1}$, J. G. Underwood ${ }^{16}$, M. Vacher ${ }^{5}$, D. J. Walke ${ }^{1}$ \\ , T. J. A. Wolf ${ }^{9}$, V. Zhaunerchyk ${ }^{2}$, J-E. Rubensson ${ }^{4}$, N.

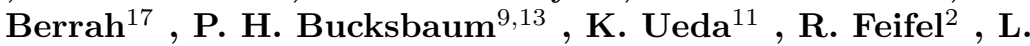 \\ J. Frasinski ${ }^{1}$ and J. P. Marangos ${ }^{1}$ \\ 1 Blackett Laboratory, Imperial College London, London SW7 2AZ, UK \\ 2 Department of Physics, University of Gothenburg, SE-412 96 Gothenburg, \\ Sweden \\ ${ }^{3}$ Institute of Theoretical Physics, Faculty of Mathematics and Physics, Charles \\ University in Prague, V Holesovickach 2, 18000 Prague, Czech Republic \\ ${ }^{4}$ Department of Physics and Astronomy, Uppsala University, SE-751 20 \\ Uppsala, Sweden \\ 5 Department of Chemistry, Imperial College London, London SW7 2AZ, \\ United Kingdom \\ ${ }^{6}$ LCLS, SLAC National Accelerator Laboratory, 2575, Sand Hill Road, Menlo \\ Park, California 94025, USA \\ 7 SOLEIL synchrotron, PLEIADES Beamline,L'Orme des Merisiers, \\ Saint-Aubin, 91192, Gif-sur-Yvette, Ile-de-France, France \\ 8 Texas Center for High Energy Density Science (CHEDS), University of Texas, \\ Austin, TX 78712 USA \\ 9 PULSE Institute, SLAC National Accelerator Laboratory, 2575, Sand Hill \\ Road, Menlo Park, California 94025, USA \\ 10 Department of Chemistry, Oxford University, Oxford OX1 3QZ, UK \\ 11 Institute of Multidisciplinary Research for Advanced Materials, Tohoku \\ University, Sendai 980-8577, Japan \\ 12 European XFEL GmbH, Albert-Einstein-Ring 19, 22761 Hamburg, Germany \\ 13 Departments of Physics and Applied Physics, Stanford University, Stanford, \\ California 94305, USA \\ 14 Theoretische Chemie, Universitta Heidelberg, Im Neuenheimer Feld 229, \\ D-69120 Heidelberg, Germany \\ 15 MAX IV Laboratory, Lund University, Box 118, 22100 Lund, Sweden \\ 16 Department of Physics and Astronomy, University College London, Gower \\ Street, London WC1E 6BT, United Kingdom \\ 17 Physics Department, Western Michigan University, Kalamazoo, Michigan \\ 49008, USA
}

$\ddagger$ Corresponding author. 
Auger electron and photoabsorption spectra of glycine in the vicinity of the oxygen K-edge measured with an X-FEL2

E-mail: t.barillot@imperial.ac.uk

Abstract. We report the first measurement of the near oxygen K-edge Auger spectrum of the glycine molecule. Our work employed an X-ray free electron laser as the photon source operated with input photon energies tunable between 527 and $547 \mathrm{eV}$. Complete electron spectra were recorded at each photon energy in the tuning range, revealing resonant and non-resonant Auger structures. Finally $a b$ initio theoretical predictions are compared whit the measured above the edge Auger spectrum and an assignment of Auger decay channels is performed. 
Auger electron and photoabsorption spectra of glycine in the vicinity of the oxygen K-edge measured with an X-FEL3

\section{Introduction}

Auger decay following core ionisation is a well-known example of a process driven by electron correlations that may occur in atoms, molecules or condensed phase matter. The process of Auger decay involves first the removal, or excitation, of an energetically deeply bound electron typically from within an atomic inner shell state. This leads to ultrafast electronic rearrangement in the atom or molecule resulting in the emission of a second electron in addition to the primary electron ionisation/excitation. There is intrinsic interest in understanding the Auger electron signature of the molecule to confirm theoretical calculations of the valence and inner valence states of the molecule and the molecular ion. Moreover, since the core electrons are energetically and spatially well localised the Auger process in a molecule can be selectively initiated by choice of photon energy close to the absorption edge of a particular atomic species within the molecule. In this way a well defined location within the molecule, determined by the position of that specific atom, can be probed by the initiation of an Auger process. Therefore it appears there is a potential path to measure charge dynamics in molecules by using Auger initiation or Auger probing.

In recent years X-ray free electron lasers have been extensively developed. This development opened the access to both high brightness and few femtosecond pulses $[1,2,3]$. The latter property permits the consideration of Auger electron signal as a route to recover charge dynamics in molecules $[4,5]$. In order to do so, one has first to determine the response of molecules to excitation in the soft X-ray range and disentangle the different electron emission channels (ie: direct valence photoemission and Auger electron emission). The high brightness and the photon energy tunability of the X-ray FEL permit to obtain an energy dependant measurement of the molecules response in a short time. Therefore, such a characterisation can be done prior to a time dependant measurement in similar experimental conditions.

The processes occurring in organic molecules are of particular interest for obvious biological reasons. Therefore glycine was chosen as it is the simplest biological molecule. Several Near Edge Absorption Fine Structure (NEXAFS) spectroscopy measurements have been realised in glycine with synchrotron radiation sources in both solid $[6,7]$ (thin layer deposition) and gas phase [8,9]. To date, however, no measured Auger spectra are reported for this system in the literature. In this article we present an Auger electron study on the glycine molecule over a range of photon energies spanning $20 \mathrm{eV}$ around the oxygen K-edge. Thus we can compare the result of excitation processes below the oxygen core ionisation energy that lead to resonant Auger electrons and the result of core ionisation giving birth to normal Auger electrons. In the latter case we can also compute the Auger spectrum relying on ab initio Algebraic Diagrammatic Construction (ADC) approach and compare the predictions to our measurements. The calculation gives us also access to the molecular orbitals contributions in the final above the edge Auger spectrum.

\section{Experimental Methods}

The experiment was conducted on an X-ray free electron laser beamline (LCLS [10], AMO endstation) providing femtosecond tuneable X-ray femtosecond pulses in SelfAmplifed Spontaneous Emission (SASE) mode around $\mathrm{E}_{\text {photon }}=537 \mathrm{eV}$ at $120 \mathrm{~Hz}$ repetition rate with a pulse energy of 50 to $550 \mu \mathrm{J}$. The photon energy was tuned by a fine scan of the electron beam energy. The range of electron bunch energy offset 
spanned from $-25 \mathrm{MeV}$ to $+25 \mathrm{MeV}$ which corresponds to a maximum offset of \pm 10 $\mathrm{eV}$ in photon energy. The electron beam energy was measured on a shot-by-shot basis by means of a beam position monitor in the final dispersive region to account for energy jitter coming from fluctuations of the electron accelerator radio frequency field. This electron bunch energy was calibrated to obtain the photon energy measuring the absorption at the $\pi^{*}$ resonance and the oxygen K-edge of a mylar filter [11]. In SASE mode a photon bandwidth of around $0.5 \%$ is expected and corresponds to an absolute bandwidth of $\sim 2.5 \mathrm{eV}$ in our experiment. X-ray pulse energy was monitored shot-byshot using fluorescence from $\mathrm{N}_{2}$ in a gas detector and tuned with a gas attenuator.

The radiation pulses interacted with a jet of neutral glycine $\left(\mathrm{NH}_{2} \mathrm{CH}_{2} \mathrm{COOH}\right)$ molecules produced in gas phase by an oven [12] at 400 K from a 99\% pure glycine sample obtained from Sigma Aldrich. With this sublimation source, we reached a target density of $\sim 10^{11}$ molecules $/ \mathrm{cm}^{3}$ in the interaction region.

Electrons created in the interaction region were collected by a $2 \mathrm{~m}$ long magnetic bottle time-of-flight spectrometer (dubbed Free Electron Laser COrrelations, FELCO) that was previously used for multi-particle correlation experiments at the LCLS [13]. This spectrometer is equipped with an MCP detector from which the signals were sent to a digitiser. The calibration of this spectrometer was obtained by measuring the $\mathrm{O}_{2}$ Auger lines. A retardation potential of $450 \mathrm{~V}$ was applied to the drift tube to optimise the resolution for electron kinetic energies between 460 to $540 \mathrm{eV}$. Although this retardation potential was mainly used to measure the Auger spectrum of glycine near the oxygen K-edge, a set of different retardation potentials (200, 300 and 380 $\mathrm{V})$ were also investigated. All the data was recorded on a shot-to-shot basis and was sorted with respect to the photon energy and normalised against pulse energy.

\section{Theoretical Methods}

For $a b$ initio calculations of the Auger decay spectra the ionisation energies were obtained using the extended second-order algebraic diagrammatic construction $[\operatorname{ADC}(2) \mathrm{x}]$ method for the singly [14] and doubly [15] ionised states. The total and partial decay widths of the core-ionised states were calculated using the Fano-ADC method [16]. Briefly, this $\mathcal{L}^{2}$ method rests on evaluation of the generalised Fano expression $[17,18]$ for the decay width, $\Gamma$, through the matrix element of the full many-electron Hamiltonian $\hat{H}$ between the bound-like $|\Phi\rangle$ and the continuum-like $\left|\chi_{\alpha, \varepsilon_{\alpha}}\right\rangle$ components of the wave function at the energy of the decaying state $E_{r}$ :

$$
\Gamma=2 \pi \sum_{\alpha=1}^{N_{c}}\left|\left\langle\Phi\left|\hat{H}-E_{r}\right| \chi_{\alpha, \varepsilon_{\alpha}}\right\rangle\right|^{2} .
$$

The summation runs over $N_{c}$ decay channels, $\varepsilon_{\alpha}$ is the kinetic energy of the emitted electron for the $\alpha$ 's decay channel. The many-electron wave functions $|\Phi\rangle$ and $\left|\chi_{\alpha, \varepsilon_{\alpha}}\right\rangle$ are obtained using the $\mathrm{ADC}(2) \mathrm{x}$ scheme for single ionisation [14].

In the center of the Fano-ADC computational procedure is the configuration selection scheme that sorts out the many-electron ADC basis states into those contributing to the expansion of the initial (bound-like) state and to the final (continuum-like) states. In the present study, we employ the energy-based configuration selection scheme [16]. As part of the Fano-ADC procedure, Stieltjes imaging $[19,20,21]$ was applied to series of increasing order block-Lanczos pseudospectra of the final state Hamiltonian until convergence with respect to the 
block-Lanczos iterations was reached [22, 23]. The calculations have been performed by using the MOLCAS quantum chemistry package [24] for Hartree-Fock selfconsistent field calculations and our own implementations of the ADC methods. For the energy calculations, aug-pCVDZ basis sets $[25,26,27]$ were used on all atomic centers. Converged Auger decay widths were obtained in aug-pCVQZ basis [25, 26, 27] without $g$ functions, uncontracted on the atomic centres with the initial core vacancy.

\section{Results and discussion}

For each photon energy the emitted electrons were recorded for kinetic energies down to $E_{\mathrm{k}} \simeq 460 \mathrm{eV}$ with the $450 \mathrm{~V}$ retardation potential. The spectrum comprises valence photoelectrons as well as Auger electrons triggered by vacancies created in oxygen core orbitals. Figure 1 presents a 2D map of the recorded electron signal where the horizontal axis corresponds to electron kinetic energy and the vertical axis corresponds to photon energy $E_{\text {photon }}$.

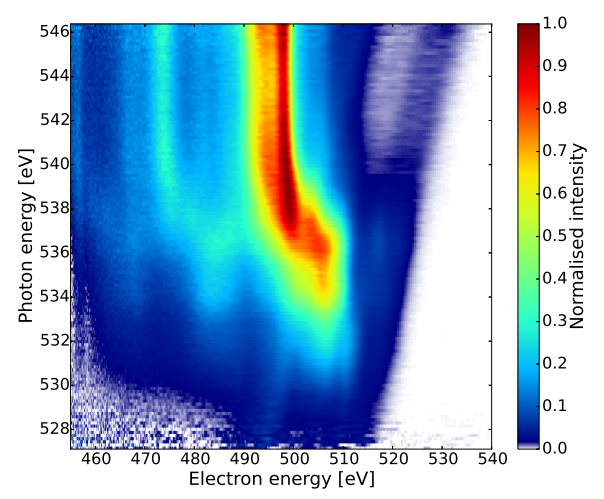

Figure 1. 2D map of the glycine emitted electron intensity for X-ray photons excitation around the oxygen K-edge.

The low intensity valence photoelectron signal is visible in the map as it drifts linearly with the photon energy. The rest of the signal corresponds to electrons emitted through Auger decay.

In the photon energy region spanning from $E_{\text {photon }}=547 \mathrm{eV}$ down to $E_{\text {photon }}=$ $539 \mathrm{eV}$ we observe normal Auger electron signal that correspond to core ionisation followed by Auger decay from the cationic to the dicatonic state. We recall here that the 1s ionisation threshold was previously estimated to be $535 \mathrm{eV}[6,7]$ in the condensed phase and $538.4 \mathrm{eV}(\mathrm{C}=\mathrm{O})$ to $540 \mathrm{eV}(\mathrm{C}-\mathrm{O}-\mathrm{H})$ in the gas phase [9]. Therefore the observed oxygen 1s ionisation potential at $539 \mathrm{eV}$ is consistent with the literature. The Auger spectrum in this photon energy region is characterised by a kinetic energy cutoff located around $E_{\mathrm{k}}=507 \mathrm{eV}$. We can extract an estimation of the double ionisation potential (DIP) of the glycine to be $32 \mathrm{eV}$. So far, only a theoretical value of $26.43 \mathrm{eV}$ for the DIP was published [28]. We also note a variation in the signal intensity with photon energy clearly identified for the brightest peak. Indeed the signal at $E_{\mathrm{k}}=500$ $\mathrm{eV}$ reaches a maximum in intensity at $E_{\text {photon }}=540 \mathrm{eV}$ then decreases with a local minimum at $E_{\text {photon }}=543 \mathrm{eV}$ and then increases again for the highest photon energy. This behaviour is clearly seen in the tail of this strongest feature (around $E_{\mathrm{k}}=495$ 
$\mathrm{eV})$. It traduces the existence of excited states corresponding to resonant transitions from the core to $\sigma$ orbitals that lie above the core ionisation limit $[8]\left(\sigma_{\mathrm{C}-\mathrm{O}-\mathrm{H}}^{*}\right.$ at 540 $\mathrm{eV}$ and $\sigma_{\mathrm{C}=\mathrm{O}}^{*}$ at $544 \mathrm{eV}$ ).

Below $E_{\text {photon }}=539 \mathrm{eV}$, electron kinetic energy cut-off dramatically increases up to $E_{\mathrm{k}}=525 \mathrm{eV}$ and slowly decreases as the photon energy decreases. The high kinetic energy cut-offs are particularly visible for the photon energy region $E_{\text {photon }}=532$ to 538 $\mathrm{eV}$ where resonant excitation processes are possibly populating excited states including the two oxygen $\pi^{*}$ resonances and the 3p Rydberg resonance $[8,9]$ although they overlap with the valence photoelectron signal. We observe that the highest kinetic energy extension of the cut-off (around $E_{\mathrm{k}}=525 \mathrm{eV}$ ) is compatible with that expected for the $\pi_{\mathrm{C}-\mathrm{O}-\mathrm{H}}^{*}$ and the Rydberg resonances (respectively 525 and $527 \mathrm{eV}$ ). The low signal associated with $\pi_{\mathrm{C}=\mathrm{O}}^{*}$ ionisation made it difficult to establish an accurate cut-off position for this ionisation channel. In this configuration, the neutral system decays through Auger emission to the cation.

Our results can be compared with previous gas phase studies on glycine by looking at the NEXAFS signal. Figure 2 exposes the total electron yield with respect to the excitation (photon) energy. The spectrum appears a lot less structured than those observed by M.L. Gordon \& al and O. Plekan \& al[8, 9], both obtained with synchrotron light source and recorded with a time of flight spectrometer at the socalled "magic angle" of $54.7^{\circ}$ with respect to the polarisation axis of the radiation. This is to be expected as the bandwidth of the SASE X-ray pulses used in the experiment reported here is large $(2.5 \mathrm{eV})$ in comparison to narrow band synchrotron pulses used by M.L. Gordon and O. Plekan. Nevertheless, the broad feature presents several underlying peaks around $E_{\mathrm{k}}=532,535$ and $537 \mathrm{eV}$. Comparing these signatures with $\pi_{\mathrm{C}=\mathrm{O}}^{*}, \pi_{\mathrm{C}-\mathrm{O}-\mathrm{H}}^{*}$ and Rydberg $3 \mathrm{p}$ resonances positions extracted from previous studies $[8,9]$ (red sticks on figure 2), we find a good agreement with our measurement. Integration over limited electron kinetic energy regions $\left(E_{\mathrm{k}}=489\right.$ to $499 \mathrm{eV}$ and $E_{\mathrm{k}}=471$ to $477 \mathrm{eV}$ ) permits to isolate the $\sigma^{*}$ resonances present at the oxygen K-edge. The corresponding curve is showed in the insert of figure 2. Although a shift of 1 to $2 \mathrm{eV}$ is observed for the $\sigma_{\mathrm{C}=\mathrm{O}}^{*}$, the position of the $\sigma_{\mathrm{C}-\mathrm{O}-\mathrm{H}}^{*}$ agrees with the literature value.

Having identified the near edge structure of glycine, we take a closer look at the Auger spectra for both resonant and non-resonant excitation. Figure 3 presents those spectra for every photon energy in $1 \mathrm{eV}$ increment and plotted on top of each other. In the range of $E_{\text {photon }}=530$ to $539 \mathrm{eV}$, where we access near edge absorption structures, the strong signal increase corresponds to resonant Auger electrons. The position of the cut-off energy extends to $E_{\mathrm{k}}=525 \mathrm{eV}$ as expected from the discussion above. The spectra exhibit three main features with maxima respectively at $E_{\mathrm{k}}=468,484$ and 506 $\mathrm{eV}$ that are clearly visible up to $E_{\text {photon }}=536 \mathrm{eV}$.

Between $E_{\text {photon }}=536 \mathrm{eV}$ and $E_{\text {photon }}=538 \mathrm{eV}$ we observe the transition of the electron spectrum from the $\pi^{*}$ resonnant spectrum to the above the edge Auger spectrum. In this region near the ionisation edge we also expect to see resonant excitation to Rydberg states. The overlap between the $\pi^{*}$ resonant signal and the above edge signal can lead to the smeared distribution we recorded. Therefore it is difficult to conclude the effect of the Rydberg resonances on the Auger electrons. Nevertheless, highly excited states such as Rydberg states must be accompanied by a high kinetic energy signal cutoff. It appears that this cutoff limit is very similar for the $\pi_{\mathrm{C}-\mathrm{O}-\mathrm{H}}^{*}$ and the Rydberg resonances but a variation of $1 \mathrm{eV}$ towards higher 
Auger electron and photoabsorption spectra of glycine in the vicinity of the oxygen K-edge measured with an X-FEL7

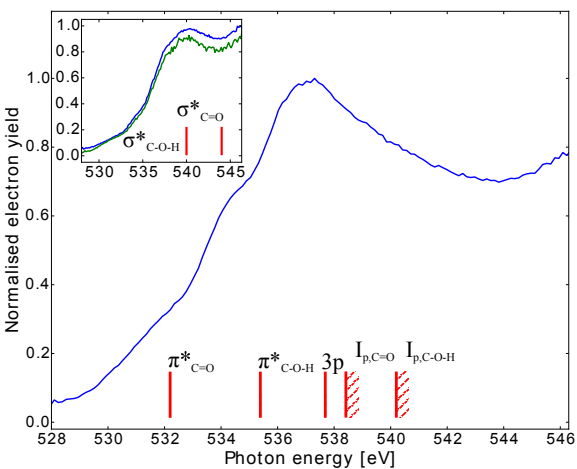

Figure 2. Photoabsorption spectrum of glycine near the oxygen K-edge obtained by integrating the total electron yield. The red lines represent the resonances positions (from left to right: $\pi_{\mathrm{C}=\mathrm{O}}^{*}, \pi_{\mathrm{C}-\mathrm{O}-\mathrm{H}}^{*}, 3 p$ ) and the shaded areas determine

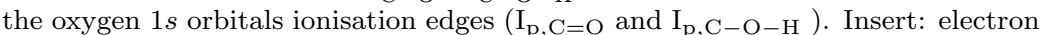
signal integrated for kinetic energies between 489 and $499 \mathrm{eV}$ (blue curve) and between 471 and $477 \mathrm{eV}$ (green curve) with position of $\sigma_{\mathrm{C}-\mathrm{O}-\mathrm{H}}^{*}$ and $\sigma_{\mathrm{C}=\mathrm{O}}^{*}$ resonances marked by vertical red lines.

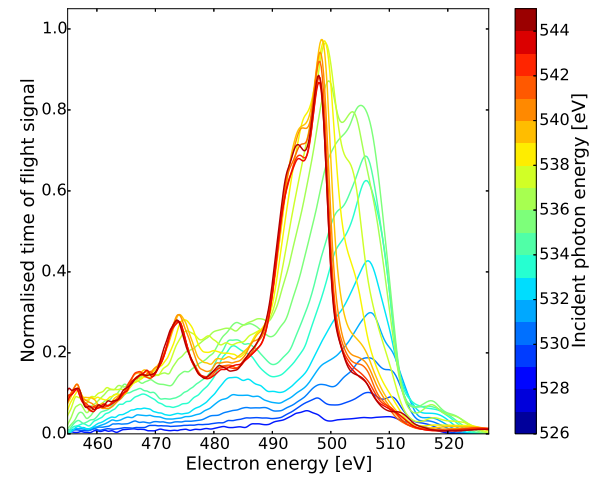

Figure 3. Superposition of Auger electrons spectra for the whole range of photon energies considered from $E_{\text {photon }}=527 \mathrm{eV}$ (dark blue curve) to $E_{\text {photon }}=545 \mathrm{eV}$ (dark red curve) with $1 \mathrm{eV}$ step.

energy for the Rydberg resonance can be seen.

At higher photon energies, the Auger structure shifts to lower kinetic energy: above $E_{\text {photon }}=539 \mathrm{eV}$, the spectrum structure does not change with photon energy and is therefore assumed to reflect the normal Auger spectrum. It exhibits three main features all shifted down by $10 \mathrm{eV}$ of kinetic energy with respect to the near edge photon energy case. It is close to the expected energy shift between the decay from the neutral to the cation and decay from the cation to the dication system (for the neutral system: $\mathrm{IP}=10 \mathrm{eV}, \mathrm{DIP}=32 \mathrm{eV}$ ). Comparing the spectra at $E_{\text {photon }}=543$ $\mathrm{eV}$ and $E_{\text {photon }}=535 \mathrm{eV}$, we note that the high photon energy spectrum exhibits more resolved structure. The apparent smearing of the resonant Auger spectra is the result of a large photon energy bandwidth for a given central frequency which, as a consequence, mixes the resonant contributions as it has already been seen in the 
photoabsorption spectrum in figure 2 .

In addition to the extensive study of the Auger spectrum near the oxygen K edge, we recorded above the carbon and nitrogen K edges Auger spectra by using lower retardation potentials on the magnetic bottle time-of-flight spectrometer for $E_{\text {photon }}=$ $545 \mathrm{eV}$. The retardation potentials used were respectively $200 \mathrm{~V}$ and $300 \mathrm{~V}$. The figures 4(a) and 4(b) present a comparison of those results with ab initio calculations. Although the experimental resolution is low due to high electron kinetic energy, there is a qualitative agreement of the Auger electron structure between the measured data and the theoretical ones.

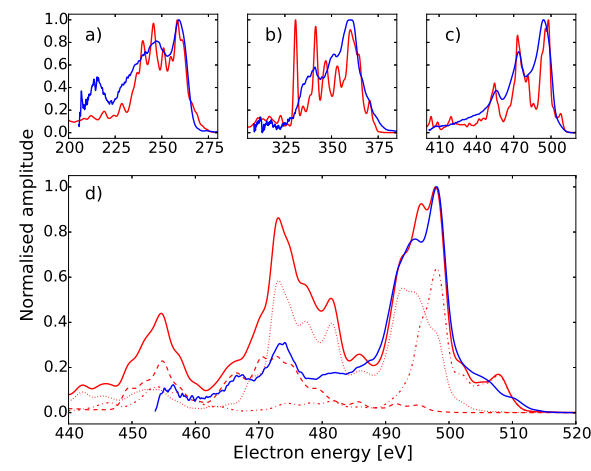

Figure 4. Comparison between theoretical (red plain curves) and experimental (blue plain curves) above the edge Auger spectra for a) carbon K-edge (200 V retardation potential), b) nitrogen $\mathrm{K}$-edge (300 $\mathrm{V}$ retardation potential), c) and d): oxygen K-edge (380 and $450 \mathrm{~V}$ retardation potential). d) presents also the dominant electronic configurations contributions to the theoretical curve: ov-ov (dotted dashed curve), iv-ov (dotted curve) and iv-iv (dashed curve).

This comparison between experimental results and calculation was also done for the above the oxygen $\mathrm{K}$ edge Auger spectrum. It is presented in the figures 4(c) and $4(\mathrm{~d})$. The contributions to the theoretical spectrum from the two oxygen atoms are summed with a $50 \%$ weight for each of them. The experimental spectra were recorded with two different retardation potentials: $380 \mathrm{~V}$ in figure $4(\mathrm{c})$ and $450 \mathrm{~V}$ in figure $4(\mathrm{~d})$. We note from figure 4(c) that the amplitudes of the three main peaks are in relative agreement with the calculation in the case of a retardation potential of $380 \mathrm{~V}$. In order to compare the Auger spectra in details, the highest retardation potential $(450 \mathrm{~V})$ was used (figure $4(\mathrm{~d})$ ). We observe that the shape of the highest kinetic energy peak is very close to the theoretical result. The amplitude difference for the second peak (centered around $E_{\mathrm{k}}=475 \mathrm{eV}$ ) and the third peak are explained by lower detection efficiency for lower electron kinetic energy in the magnetic bottle due to the presence of the strong retardation potential. The present calculation presents a DIP of $25.0 \mathrm{eV}$ closer to the Density Functional Theory (DFT) results [28] than the experimental one. Nevertheless the oxygen core single ionisation potentials are $536.8 \mathrm{eV}$ and $538.8 \mathrm{eV}$ for $\mathrm{O}_{\mathrm{C}=\mathrm{O}}$ and $\mathrm{O}_{\mathrm{C}-\mathrm{O}-\mathrm{H}}$ respectively which are close to the experimental ones. It also appears that the Auger relaxation does not populate the ground state of the dication but a state lying at $30.6 \mathrm{eV}$ above the ground state of the neutral glycine. This explain the relatively good agreement between the theoretical predictions and the experiment as the latter value can be compared to the $32 \mathrm{eV}$ value 
extracted from measurements. Moreover, the use of an oven as source of molecular jet implies the existence of different isomers (glycine I and glycine III). In contrast the calculation takes into account only the glycine I isomer but no noticeable differences arise.

The theoretical spectrum is decomposed in the different electronic configurations participating to the Auger decay channels (figure 4(d)): outer valence to core orbital recombination accompanied by outer valence electron emission (ov-ov), inner valence to core recombination with outer valence electron emission (iv-ov) and inner valence to core recombination with inner valence electron emission (iv-iv). The distinction between inner valence molecular orbitals (6a' to 10a') and outer valence orbitals (11a' to $\left.20 \mathrm{a}^{\prime}\right)$ is done considering their underlying $2 \mathrm{~s}$ or $2 \mathrm{p}$ atomic character. Nevertheless, all those orbitals are delocalised over the entire molecule. We observe that the highest kinetic energy feature around $495 \mathrm{eV}$ is mainly composed by electrons emitted through channels (ov-ov) and (iv-ov). The second structure $\left(E_{\mathrm{k}}=475 \mathrm{eV}\right)$ is essentially the result of inner valence to core recombination with two main emission configurations: (iv-ov) and (ov-ov). Interestingly, the third band $\left(E_{\mathrm{k}}=455 \mathrm{eV}\right)$ is composed by the three possible configurations in relatively equal proportions. The presence of the (ov-ov) configuration at this low energy stresses the extremely mixed character of corresponding dicationic states and is the illustration of the breaking of the molecular orbital picture to represent this Auger decay channel.

\section{Conclusion}

In this article, we performed the first measurement of Auger spectrum in glycine. We have demonstrated the use of X-ray FEL pulses for the investigation of Auger processes occurring near the oxygen $\mathrm{K}$ edge of a small organic molecule (glycine) in the gas phase. We recorded Auger spectra as a function of photon energy, following their evolution through resonant and non resonant processes. Thus we obtained a full picture on the evolution of the Auger spectra. In our experiment we scanned the SASE FEL photon energy by scanning the electron energy which was found to have a number of advantages. The high photon flux allows the electron spectrum to be rapidly accumulated despite the relatively low sample density allowing the total scan time in these measurements to be within 10 minutes. This paves the way for future time resolved experiments in the pump-probe mode where the time for accumulation of data at each value of photon energy and time delay should be short to allow complete measurements to be acquired in a few hours. Further measurements with narrow band sources, however, would be desirable to fully resolve the near edge structures and the details of the resonant Auger spectra in the glycine molecule.

Finally the above the edge Auger spectrum has been compared with ab-initio calculation, revealing a good agreement between experiment and theory for the glycine I isomer. The theory also allowed to determine the dominant configuration in the decay channels constituting the above oxygen K-edge Auger spectrum of glycine.

\section{Acknowledgments}

The Authors would like to thank the SLAC support and in particular C. O'Grady, A. Mitra and M. J. Bucher. This work was supported financially by the Engineering and Physical Sciences Research Council (UK) (EPSRC) grant EP/I032517/1, the ERC ASTEX project 290467 and the EPSRC grant EP/M023729/1. A.S. acknowledges 
the STFC for financial support. P.K. acknowledges financial support by the Czech Science foundation (Project GACR P208/12/0521). H.F., K.M. and K.U. are grateful to support by the X-ray Free Electron Laser Priority Strategy Program of MEXT and by Tagen project by IMRAM. This work has been financially supported by the Swedish Research Council (V.R.) and the Knut and Alice Wallenberg Foundation, Sweden. V.Z. acknowledges the financial support from the Swedish FEL center. The experimental research was carried out at the Linac Coherent Light Source (LCLS) at the SLAC National Accelerator Laboratory. LCLS is an Office of Science User Facility operated for the US Department of Energy Office of Science by Stanford University

\section{References}

[1] Lutman A A, Coffee R, Ding Y, Huang Z, Krzywinski J, Maxwell T, Messershmidt M and Nuhn H -D 2013 Phys. Rev. Lett. 110134801

[2] McNeil B W J and Thompson N R 2010 Nat. Photon. 4 814-21

[3] Schulz S, et al. 2015 Nat. Commun. 65938

[4] Cooper B, Kolorenč P, Frasinski L J, Averbukh V and Marangos J P 2014 Faraday Discussions $17193-111$

[5] McFarland B K et al. 2014 Nat Commun. 54235

[6] Tanaka M, Nakagawa K, Koketsu T, Agui A and Yokoya A 2001 J. Synchrotron Rad. 8 1009-11

[7] Zubavichus Y, Zharnikov M, Schaporenko A and Grunze M 2004 J. of Electron Spectroscopy and Related Phenomena 134 25-33

[8] Gordon M L, Cooper G, Morin C, Araki T, Turci C C, Kaznatcheev K and Hitchcock A P 2003 J. Phys. Chem. A $1076144-59$

[9] Plekan O, Feyer V, Richter R, Coreno M, de Simone M, Prince K C and Carravetta V 2007 J. of Electron Spectroscopy and Related Phenomena 155 47-53

[10] Emma P, et al 2010 Nat. Photon. 4 641-47

[11] Rightor E G, Hitchcock A P, Ade H, Leapman R D, Urquhart S G, Smith A P, Mitchell G, Fischer D, Shin H J and Warwick T 1997 J. Phys. Chem. B 101 1950-60

[12] McFarland B K, et al 2014 J. of Physics: Conference Series 488012015

[13] Frasinski L J, et al 2013 Phys. Rev. Lett. 111073002

[14] Schirmer J, Cederbaum L S and Walter O 1983 Phys. Rev. A 281237

[15] Schirmer J and Barth A 1984 Z. Phys. A 317267

[16] Averbukh V and Cederbaum L S 2005 J. Chem. Phys. 123204107

[17] Fano U 1961 Phys. Rev. 1241866

[18] Åberg T and Howat G 1982 Handbuch der Physik vol. 31 ed W. Mehlhorn W (Springer, Berlin)

[19] Langhoff P W 1973 Chem. Phys. Lett. 2260

[20] Langhoff P W, Corcoran C T, Sims J S, Weinhold F and Glover R M 1976 Phys. Rev. A 14 1042

[21] Langhoff P W 1979 Electron-Molecule and Photon-Molecule Collisions ed Rescigno T, McKoy $\mathrm{V}$ and Schneider B (Plenum, New York).

[22] Kopelke S, Gokhberg K, Averbukh V, Tarantelli F and Cederbaum L S 2011 J. Chem. Phys. 134094107

[23] Kopelke S, Gokhberg K, Cederbaum L S, Tarantelli F and Averbukh V 2011 ibid 134024106

[24] Karlström G, Lindh R, Malmqvist P - $\AA$, Roos B O, Ryde U, Veryazov V, Widmark P -O, Cossi M, Schimmelpfennig B, Neogrady P and Seijo L 2003 Comp. Mat. Sci. 28222

[25] Dunning T H Jr. 1989 J. Chem. Phys. 901007

[26] Schuchardt K L, Didier B T, Elsethagen T, Sun L, Gurumoorthi V, Chase J, Li J, and Windus T L 2007 J. Chem. Inf. Model. 47(3) 1045

[27] D. Feller 1996 J. Comp. Chem. 17(13), 1571

[28] S. Maclot, et al 2013 J. Phys. Chem. Lett. 4 3903-09 